\title{
Redshift of excitons in carbon nanotubes caused by the environment polarizability
}

\author{
Michael Rohlfing* \\ Fachbereich Physik, Universität Osnabrück, Germany
}

(Dated: August 17, 2018)

\begin{abstract}
Optical excitations of molecular systems can be modified by their physical environment. We analyze the underlying mechanisms within many-body perturbation theory, which is particularly suited to study non-local polarizability effects on the electronic structure. Here we focus on the example of a semiconducting carbon nanotube, which observes redshifts of its excitons when the tube is touched by another nanotube or other physisorbates. We show that the redshifts mostly result from the polarizability of the attached ad-system. Electronic coupling may enhance the redshifts, but depends very sensitively on the structural details of the contact.
\end{abstract}

With improving precision of nanostructured sample preparation, controlled manipulation of optical excitations on the atomic scale becomes possible, opening a path to nanometer photonics in anorganic, organic, and pure-carbon semiconductor systems. One prototypical material is given by carbon nanotubes (CNT) and their excitons $[1-10]$. Here the influence of the surrounding physical environment is of high importance for the stability and control of excited states. In this Letter we discuss (by many-body perturbation theory, MBPT [11, 12]) two mechanisms by which excitonic states in CNT and in other nano-scale semiconducting systems can be manipulated by surrounding material.

In order to demonstrate the effects, we consider systems that have recently been investigated experimentally. The optical excitations of a CNT exhibit redshifts to lower energy when the CNT is put in nitrogen [6] or when it is touched by another CNT 7]. To our knowledge, a comprehensive understanding and analysis of these effects is still missing, hindering their systematic and widespread investigation. We study these issues within MBPT [11, 12], which has become the standard approach to CNT excitons [3-5, 10]. We include the physical environment (here: another CNT or nitrogen), thus going far beyond those previous MBPT studies in which the CNT were considered in vacuum. We believe that our approach and conclusions apply to many other materials, as well.

The spectrum of one system (1) might be shifted in energy by various mechanisms when contacted with another system (2). We focus on two mechanisms which could be most relevant for chemically inert semiconducting systems: (i) modification of the exciton energy in system 1 by electronic polarization of system 2, and (ii) electronic coupling between systems 1 and 2 . We show below that the second effect (ii) depends heavily on the details of the contact geometry, thus requiring sample perfection that may be too difficult to achieve for CNT. The redshifts from the polarizability effect (i), on the other hand, are much more robust and may fully explain the experimental findings [6, 7]. Throughout the paper we focus on electronic polarizability, i.e. nuclear motion or phonons

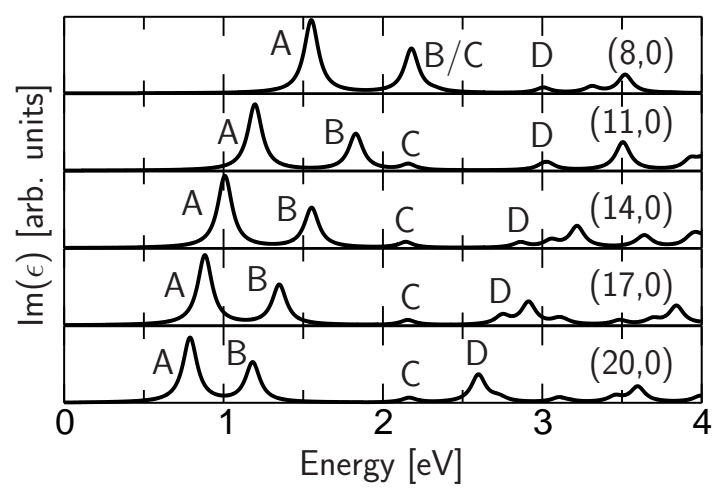

FIG. 1: Optical spectra of isolated $(N, 0)$ CNT (for the electric field of the light along the tube). Characteristic peaks are denoted as A, B, C, and D (cf. Fig. 2 d,e).

and corresponding polarization are not considered. Also, spectral shifts from changes of the intrinsic structure of the CNT (e.g., deformation or chemical modification) are not considered in this paper, assuming that physisorption of inert material leaves the CNT structure unaffected.

Both the fundamental gap and the excitonic binding energy are reduced when system 1 is contacted with other polarizable material [17 21]. However, a homogeneous change of the dielectric background would change both quantities by the same amount (to lowest order), leaving the optical excitations unchanged. Instead, the shifting of optical excitations requires additional polarizability with spatial inhomogeneity in closest vicinity of system 1 (e.g. substrate surfaces, neighboring molecules, etc.). In some cases, such additional polarizability might be described as a continuous medium with a (flat or curved) surface, allowing to express its effects on system 1 by analytical expressions (e.g., solvent models or image-potential effects) 21]. However, it is not clear if such an approach applies to the highly anisotropic and inhomogeneous polarizability of an adjacent CNT. Instead we include the additional polarizability with atomistic resolution in our MBPT approach [16].

Two technical specifications should be mentioned: (i) 
We mostly employ a simplified "LDA $+G d W$ " version of MBPT [16, 17]. While being somewhat less accurate (on an absolute energy scale) than a full GW/BSE calculation with RPA dielectric screening, LDA $+G d W$ still fully incorporates all relevant aspects of the screening (atomistic resolution, local-field effects, and nonlocality). Our reference calculations within the conventional $G W / \mathrm{BSE} / \mathrm{RPA}$ approach confirms the applicability of LDA $+G d W$ (see below). (ii) Both in the GWA and the BSE part of LDA $+G d W$, we employ the same $\mathbf{k}$-point sampling (16 $\mathbf{k}_{i}$-points from the first Brillouin zone, including the $\Gamma$ point) for representing the screened Coulomb interaction $W$. Since each $\mathbf{k}_{i}$ represents a subvolume $V_{i}$ of reciprocal space, we replace $W\left(\mathbf{k}_{i}\right)$ by its average in $V_{i}$, i.e. by $1 / V_{i} \int_{V_{i}} W(\mathbf{k}) d^{3} k$. This guarantees the aforementioned cancelation of gap reduction and electron-hole interaction reduction when the dielectric background is changed or when polarizability is added at large distance from the CNT.

Optical spectra for individual $(N, 0)$ CNT $(N=8-20)$ are shown in Fig. 1] For the $(8,0) \mathrm{CNT}$, the first four optically active excitations (A-D) are found at $1.55 \mathrm{eV}$, $2.18 \mathrm{eV}, 2.33 \mathrm{eV}$, and at $3.01 \mathrm{eV}$. For the larger tubes, peaks $\mathrm{A}$ and $\mathrm{B}$ move to lower excitation energy while $\mathrm{C}$ and $\mathrm{D}$ remain in the visible range. These $\mathrm{LDA}+G d W$ results (for the $(8,0) \mathrm{CNT}$ ) differ slightly from our full GW/BSE/RPA reference calculation, which yields 1.60 $\mathrm{eV}, 2.05 \mathrm{eV}, 2.42 \mathrm{eV}$ and $3.16 \mathrm{eV}$ for peaks A-D. A previous GW/BSE/RPA calculation [3] yielded $1.55 \mathrm{eV}$ and $1.80 \mathrm{eV}$ in comparison with experimental data of $1.60 \mathrm{eV}$ and $1.88 \mathrm{eV}$ [1, 2]. The slight deviations of our LDA $+G d W$ data result from the approximations involved and from the employment of a model screening. The screening [16] is based on the static RPA polarizabilites of the carbon atoms (neglecting local-field effects) which amount to 7-7.8 $\AA^{3}$ (depending on $N$ ) in the direction along the tube axis, 5.5-6.7 $\AA^{3}$ along the tube surface but perpendicular to the axis, and 1.1-1.2 $\AA^{3}$ in radial direction. Starting from these values, $\epsilon_{\mathbf{G}, \mathbf{G}^{\prime}}(\mathbf{q}, \omega)$ fully incorporates inhomogeneity, non-locality and localfield effects [16].

Starting from the spectra of Fig. 1, we now include in the screening the polarizability of system 2. Fig. 2 a shows the effect on a $(8,0) \mathrm{CNT}$ when another $(8,0)$ tube is attached to it (at a distance of $3.15 \AA$ ). All peaks are redshifted to lower excitation energy. Note that the redshifts are significantly smaller than the reduction of the fundamental gap and of the exciton binding energy (both $\sim 0.3 \mathrm{eV}$ ). Both effects largely cancel each other, yielding only a small net effect. Our full GW/BSE/RPA reference calculation yields the same redshifts to within $10 \mathrm{meV}$.

The redshift results from the polarization of CNT 2 when an exciton on CNT 1 is excited [21]. As illustration, Fig. $2 \mathrm{~b}$ shows the change of electronic charge $\left[\Delta \rho^{[E x c]}(\mathbf{r}):=\rho_{v}(\mathbf{r})-\rho_{c}(\mathbf{r})\right]$ when exciton $\mathrm{D}$ is excited
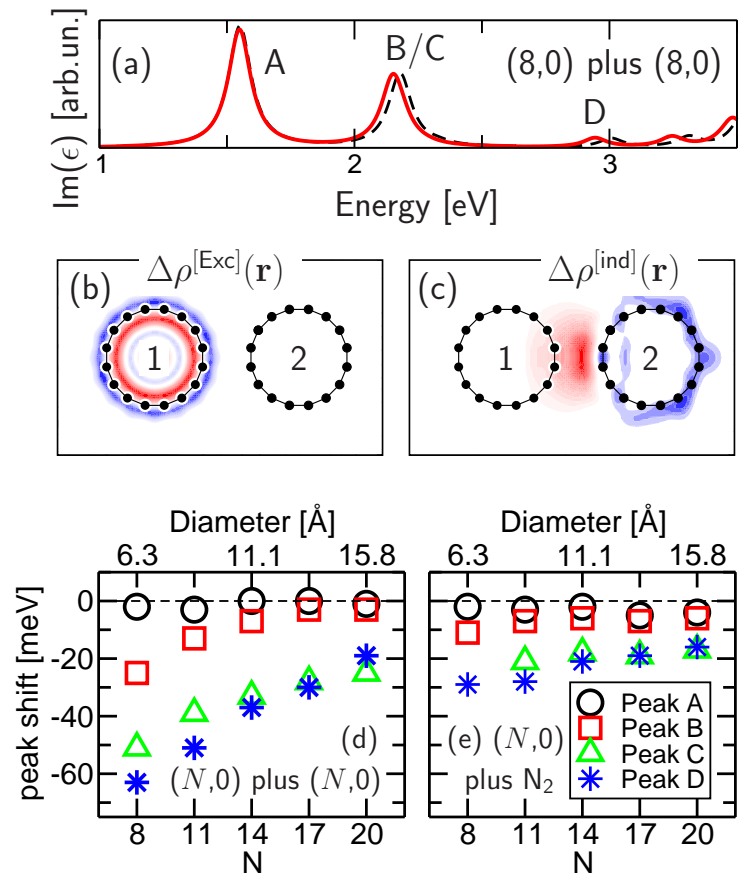

FIG. 2: Effect of additional polarizability on the optical spectrum of a single CNT, disregarding electronic coupling. (a) Spectrum of a $(8,0)$ CNT while in contact with another $(8,0)$ CNT. The isolated $(8,0)$ CNT spectrum (Fig. 1a) is included as dashed curve. (b) Charge distribution $\left[\Delta \rho^{[E x c]}(\mathbf{r}):=\rho_{v}(\mathbf{r})-\rho_{c}(\mathbf{r})\right]$ of exciton D from panel a (blue: negative charge, red: positive charge). (c) Induced charge distribution on the other CNT (blue: negative charge, red: positive charge). (d) Redshift of spectral peaks A-D of a $(N, 0) \mathrm{CNT}$ when in contact with another $(N, 0)$ CNT (i.e., two equal CNT). (e) Redshift of spectral peaks A-D of a $(N, 0)$ $\mathrm{CNT}$ when being covered by $\mathrm{N}_{2}$ molecules [State $\mathrm{C}$ cannot be identified for $N=8]$.

on CNT 1. Since the conduction $(c)$ states are closer to the vacuum level than the valence $(v)$ states, the former extend farther into the vacuum, causing $\Delta \rho(\mathbf{r})$ to be slightly positive inside CNT 1 and slightly negative outside. This slight inhomogeneous charge distribution of the exciton leads to a polarization of the material nearby (here: CNT 2), as shown in Fig. 2 c [induced charge density $\left.\Delta \rho^{[\text {ind }]}(\mathbf{r})\right]$. The interaction between $\Delta \rho^{[E x c]}(\mathbf{r})$ and $\Delta \rho^{[\text {ind] }}(\mathbf{r})$ finally redshifts the excitation.

Note that such effects are particularly important if $\Delta \rho^{[E x c]}(\mathbf{r})$ is non-zero at such positions $\mathbf{r}$ where system 2 has high charge susceptibility (caused by its own electronic structure) and inhomogeneity. This is mostly the case at distances of about 1-3 $\AA$ from the nuclei of system 2 . Here system 2 can be polarized by $\Delta \rho^{[E x c]}(\mathbf{r})$ even if it carries no dipole. For any exciton, $\Delta \rho^{[E x c]}(\mathbf{r})$ must be non-zero somewhere (if not simply for the abovementioned argument that electrons extend farther into vacuum than holes). The effect described here should thus be of widespread relevance. 
Since the range of the susceptibility of system 2 amounts to a few $\AA$, only, the redshifts are quite sensitive to the distance between systems 1 and 2 . We find that they decrease by $50 \%$ when the distance between CNT 1 and CNT 2 increases by $1 \AA$. Significant redshifts thus require physisorption distance. This would be different for excitons with charge-transfer dipole, for which the polarizability effects are long-ranged and are generally stronger [21].

From Figs. 2 b and $\mathrm{c}$ it is clear that the polarizability only affects a few atoms of the CNT, i.e. in the immediate contact between system 1 and 2. Since the excitons are equally distributed around the tube, the amplitude of $\Delta \rho^{[E x c]}(\mathbf{r})$ at each atom scales like $1 / N$. An exciton of a larger-diameter CNT thus causes weaker polarization of CNT 2 and observes smaller redshifts, as can be seen in our data for all five $(N, 0)$ CNT (each contacted with another $(N, 0) \mathrm{CNT})$ in Fig. 2 d. Wang et al. measured redshifts of 30-50 meV [7] (in the visible spectrum) for CNT with diameter of $18-19 \AA$ (maybe about $15 \AA$ in our notation of nucleus-to-nucleus distances). Our results at that diameter are comparable to these data (although slightly smaller).

Effects independent of CNT diameter can be expected when the entire surface is covered by polarizable adsorbates. In the experiments by Finnie et al. [6] physisorbed $\mathrm{N}_{2}$ was the most likely explanation for the observed redshift of $20-30 \mathrm{meV}[6]$. We find that $\mathrm{N}_{2}$ physisorbs on graphene (flat-lying at a distance of $\sim 3 \AA$, slightly depending on the adsorption site) with a DFTLDA binding energy of $\sim 80 \mathrm{meV}$ (in DFT-LDA). We have included $\mathrm{N}_{2}$ molecules in the CNT calculations. The physisorption on the CNT was modeled by a LennardJones potential based on our $\mathrm{N}_{2}$-graphene DFT-LDA results, complemented by an $\mathrm{N}_{2}-\mathrm{N}_{2}$ intermolecular potential [22]. Room-temperature molecular dynamics (MD) then yields realistic structures with the entire CNT surface covered by $\mathrm{N}_{2}$. Subsequent LDA- $G d W$ calculations (averaged over $10 \mathrm{MD}$ snap shots) yield the redshifts shown in Fig. 2 e. The physisorption of $\mathrm{N}_{2}$ does in fact yield redshifts that depend much less on tube diameter. However, the polarizability of $\mathrm{N}_{2}$ is much smaller than that of an adjacent CNT, leading to significantly weaker redshift effects.

So far, the electronic degrees of freedom of the adjacent object were not considered. This is certainly valid for the physisorption of $\mathrm{N}_{2}$, which has HOMO (LUMO) states far below (above) those of the CNT. For the coupling between two CNT, on the other hand, significant inter-tube interaction among electrons and holes is expected, calling for calculations in which CNT 2 is fully included (not only its polarizability). It turns out that (i) for some of the excitons the effects are much more drastic than the effects from the polarizability alone, and (ii) the coupling depends very sensitively on the contact geometry. Fig. 3 $\mathrm{a}+\mathrm{b}$ shows spectra for a pair of $(8,0) \mathrm{CNT}$ in two differ-
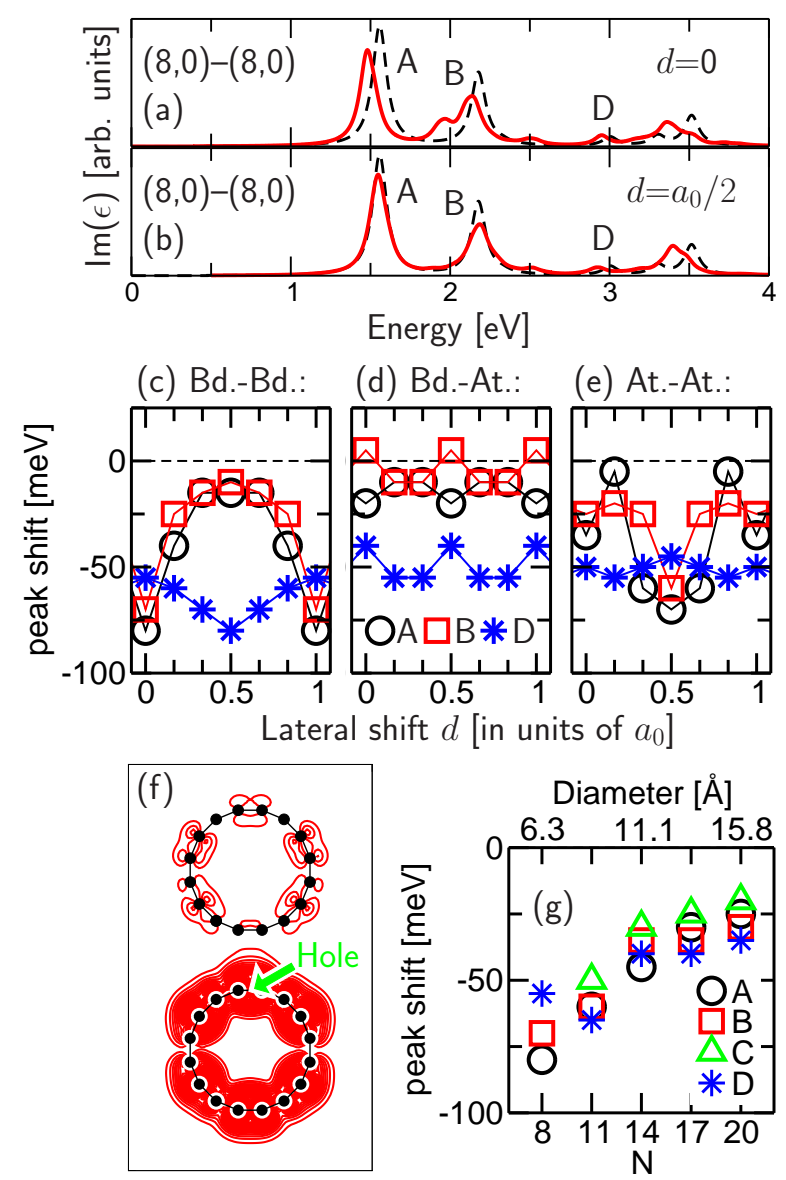

FIG. 3: Effect of electronic coupling between two CNT on their spectra. (a,b) Spectrum of two coupled $(8,0)$ CNT for two contact structures (Bond-Bond configuration with $d=0$ and $d=a_{0} / 2$; cf. panel c). (c-e) Redshift of the spectral peaks $\mathrm{A}, \mathrm{B}$, and D of two coupled $(8,0) \mathrm{CNT}$, depending on the contact structure. [Peak $\mathrm{C}$ cannot be identified in many of the spectra for $N=8$.] (f) Electron distribution (relative to the hole) for exciton A of panel a. (g) Redshift of A-D of a $(N, 0)$ CNT when in contact with another $(N, 0)$ CNT (all in BondBond configuration with $d=0$, cf. panel a).

ent configurations. In both structures, bonds of the two CNT face each other (cf. Fig. 2 b $+c$ and Fig. 3 f), but for panel $\mathrm{b}$ tube 2 was laterally shifted by half a lattice constant $\left(d=a_{0} / 2=2.13 \AA\right)$, leading to completely different redshifts. The dependence of the redshifts on the contact geometry is summarized in Fig. 3 c-e. In addition to the lateral shift $d$, CNT 2 was rotated by $11.25^{\circ}$ for panel $\mathrm{d}$, such that bonds of CNT 1 face atoms on CNT 2 ("BondAtom"). For panel e, both tubes were rotated by $11.25^{\circ}$ such that atoms face atoms along the connection line between the tubes ("Atom-Atom"). The resulting redshifts of peaks $\mathrm{A}$ and $\mathrm{B}$ range from $-80 \mathrm{meV}$ to zero. They are extremely sensitive to the slightest rotation or shift of the tubes, which strongly change the coupling-matrix elements between the atomic wave-function components 
(that are highly sensitive to the orientation of the atoms to each other). Peak D, on the other hand, always shows a redshift of about $-60 \mathrm{meV}$ already observed in Fig. 2. i.e. no significant shift beyond the polarizability effect (which is, of course, automatically included). Such high sensitivity is not at all found for the polarizability effect (Fig. 2), which is the same (to within $1 \mathrm{meV}$ ) for all rotational angles and shifts. Apparently, the polarizability effect is much more robust than electronic coupling.

This high sensitivity of the electronic coupling raises the question if this effect would be relevant for experimental observations, like Ref. [7]. Exactly the same contact geometry would be required over many lattice constants, which seem unlikely. Even the basic requirement of having two CNT with identical chirality (and thus lattice constant) may not be fulfilled. Slightly different lattice constants of the two CNT and consequently noncommensurable contact geometry would immediately reduce the electronic coupling effect. We therefore believe that mainly the polarizability effects as discussed before can be held responsible for the measured redshifts. However, a slight increase (on average) of the redshifts might result from the electronic coupling.

Nonetheless a closer analysis of the electronic coupling is helpful and may guide further studies in which the required perfect contact quality can be achieved. In particular, the mere size of redshifts of up to $80 \mathrm{meV}$ (in addition to the polarizability effect) is interesting. At first glance the interaction between two identical excitons $\left|\Psi_{1}\right\rangle$ and $\left|\Psi_{2}\right\rangle$ on identical CNT 1 and 2 should mainly lead to superpositions $\left|\Psi_{1}\right\rangle \pm\left|\Psi_{2}\right\rangle$ with only slight changes of the excitation energy; furthermore, the energy shifts should be more or less symmetric to the red and the blue (however, one of the coupled excitons might get the full dipole strength and the other one become dark). In fact, our analysis of test states like $\left|\Psi_{1}\right\rangle \pm\left|\Psi_{2}\right\rangle$ do show very small spectral shifts for peaks $\mathrm{A}$ and $\mathrm{B}$. The significant redshift of A and B (for some geometries) results from a totally different effect, i.e. a spatial spillout of the excitons across the CNT contact. Schematically, $\left|\Psi_{1}\right\rangle \hat{=}|v 1 \rightarrow c 1\rangle$ is composed from holes $(v)$ and electrons $(c)$ on CNT $1\left(\left|\Psi_{2}\right\rangle \hat{=}|v 2 \rightarrow c 2\rangle\right.$ on CNT 2, respectively). Electronic coupling adds charge-transfer contributions $|v 1 \rightarrow c 2\rangle$ and $|v 2 \rightarrow c 1\rangle$ (which are not contained in states like $\left|\Psi_{1}\right\rangle \pm\left|\Psi_{2}\right\rangle$ ), thus forming an exciplex state (similar to excimer states among atoms or molecules).

The exciplex nature can be visualized by the electronhole correlation function, e.g. Fig. 3 f. For the hole on CNT 1, the electron is mostly found on the same tube but also has significant amplitude (about 10\%) on CNT 2 (from the $|v 1 \rightarrow c 2\rangle$ contribution). The admixture of such charge-transfer contribution constitutes a reduced quantum confinement (QC) between electron and hole across the tubes, compared to the $\mathrm{QC}$ on a single tube. QC tends to increase excitation energies (e.g., in semiconductors); here we find the reversed effect of reduced excitation energy (i.e., redshift) due to reduced QC. [In excimers this effect is responsible for significant interatomic binding.] Similar to the size dependence of the polarizability effect (Fig. 2 d), this exciplex redshift becomes smaller for the larger CNT, as shown in Fig. $3 \mathrm{~g}$ (all for Bond-Bond contacts with $d=0$ ).

In conclusion, we have shown that electronic polarizability of neighboring systems can redshift exciton states of carbon nanotubes. Here the exciton's charge-density distribution induces charge density in the neighboring system. This mechanism is particularly effective when the excited system is very close to the neighboring system, e.g. at physisorption distance. This should be relevant not only for carbon nanotubes (which were taken as an example in the present study), but also for other molecules, polymers, etc. in contact with chemically inert systems that exhibit electronic polarizability. In addition to the polarizability effect, electronic coupling between the systems can significantly enhance the redshifts. However, very precise control of the contact structure would be required for electronic coupling, since it depends very sensitively on the atom positions of the two components relative to each other. If the contact is disturbed by periodicity mismatch, thermal fluctiation, or other uncontrolled features, electronic coupling might be immediately destroyed. The polarizability effect, on the other hand, is very robust with respect to contact details. Here we investigated both mechanisms within many-body perturbation theory, which allows to address both the non-local polarizability effect and electronic coupling.

We thank the John von Neumann Institut für Computing at FZ Jülich for computational resources.

* Electronic address: Michael.Rohlfing@uos.de

[1] S.M. Bachilo et al., Science 298, 2361 (c022002).

[2] R.B. Weisman and S.M. Bachilo, Nano Lett. 3, 1235 (2003).

[3] C.D. Spataru et al., Phys. Rev. Lett. 92, 077402 (2004).

[4] E. Chang et al., Phys. Rev. Lett. 92, 196401 (2004).

[5] J. Maultzsch et al., Phys. Rev. B 72, 241402 (2005).

[6] P. Finnie et al., Phys. Rev. Lett. 94, 247401 (2005).

[7] F. Wang et al., Phys. Rev. Lett. 96, 167401 (2006).

[8] P. Avouris et al., Nature Nanotech. 2, 605 (2007).

[9] I.V. Bondarev et al., Phys. Rev. B 80, 085407 (2009).

[10] C.D. Spataru and F. Leonard, Phys. Rev. Lett. 104, 177402 (2010).

[11] G. Onida et al., Rev. Mod. Phys. 74, 601 (2002).

[12] M. Rohlfing and S.G. Louie, Phys. Rev. B 62, 4927 (2000).

[13] C.S. Wang and W.E. Pickett, Phys. Rev. Lett. 51, 597 (1983);

[14] F. Gygi and A. Baldereschi, Phys. Rev. Lett. 62, 2160 (1989).

[15] V. Fiorentini and A. Baldereschi, Phys. Rev. B 51, 17 196 (1995). 
[16] M. Rohlfing, Phys. Rev. B 82, 205127 (2010).

[17] A. Greuling et al., Phys. Rev. B 84, in print (2011).

[18] M. Rohlfing et al., Phys. Rev. Lett. 91, 256802 (2003).

[19] J.B. Neaton et al., Phys. Rev. Lett. 97, 216405 (2006).

[20] K.S. Thygesen and A. Rubio, Phys. Rev. Lett. 102,
046802 (2009).

[21] J.M. Garcia-Lastra and K.S. Thygesen, Phys. Rev. Lett. 106, 187402 (2011).

[22] G. Galassi and D.J. Tildesley, Mol. Sim. 13, 11 (1994). 